\title{
Reconstruction of Black Hole Metric Perturbations from Weyl Curvature II: The Regge-Wheeler gauge
}

\author{
Carlos O. Lousto \\ Department of Physics and Astronomy, and Center for Gravitational Wave \\ Astronomy, The University of Texas at Brownsville, Brownsville, Texas 78520, USA
}

\begin{abstract}
Perturbation theory of rotating black holes is described in terms of the Weyl scalars $\psi_{4}$ and $\psi_{0}$; each satisfying the Teukolsky's complex master wave equation with spin $s=\mp 2$, and respectively representing outgoing and ingoing radiation. We explicitly construct the metric perturbations out of these Weyl scalars in the ReggeWheeler gauge in the nonrotating limit. We propose a generalization of the ReggeWheeler gauge for Kerr background in the Newman-Penrose language, and discuss the approach for building up the perturbed spacetime of a rotating black hole. We also provide both-way relationships between waveforms defined in the metric and curvature approaches in the time domain, also known as the (inverse-) Chandrasekhar transformations, generalized to include matter.

PACS numbers: 04.25.Nx, 04.30.Db, 04.70.Bw
\end{abstract}

\section{Introduction}

There is a formulation of the perturbation problem derived from the Newman-Penrose formalism [20] that is valid for perturbations of rotating black holes. 225] This formulation fully exploits the null structure of black holes to decouple the curvature perturbation equations into a single master wave equation that, in Boyer-Lindquist coordinates $(t, r, \theta, \varphi)$, can be written as:

$$
\begin{aligned}
& \left\{\left[a^{2} \sin ^{2} \theta-\frac{\left(r^{2}+a^{2}\right)^{2}}{\Delta}\right] \partial_{t t}-\frac{4 M a r}{\Delta} \partial_{t \varphi}-2 s\left[(r+i a \cos \theta)-\frac{M\left(r^{2}-a^{2}\right)}{\Delta}\right] \partial_{t}\right. \\
& +\Delta^{-s} \partial_{r}\left(\Delta^{s+1} \partial_{r}\right)+\frac{1}{\sin \theta} \partial_{\theta}\left(\sin \theta \partial_{\theta}\right)+\left(\frac{1}{\sin ^{2} \theta}-\frac{a^{2}}{\Delta}\right) \partial_{\varphi \varphi} \\
& \left.+2 s\left[\frac{a(r-M)}{\Delta}+\frac{i \cos \theta}{\sin ^{2} \theta}\right] \partial_{\varphi}-\left(s^{2} \cot ^{2} \theta-s\right)\right\} \Psi=4 \pi \Sigma T
\end{aligned}
$$

where $M$ is the mass of the black hole, $a$ its angular momentum per unit mass, $s$ the spin of the perturbation, $\Sigma \equiv r^{2}+a^{2} \cos ^{2} \theta$, and $\Delta \equiv r^{2}-2 M r+a^{2}$. The source term $T$ is built up from the energy-momentum tensor [25. Gravitational perturbations, corresponding to $s= \pm 2$, are compactly described in terms of contractions of the Weyl tensor with a null tetrad. The components of the tetrad (also given in Ref. [25]) are appropriately 
chosen along the repeated principal null directions of the background spacetime [see Eq. (3) below]. The resulting (infinitesimal) coordinate and tetrad invariant components of the Weyl curvature are given by

$$
\Psi= \begin{cases}\rho_{K}^{-4} \psi_{4} \equiv-\rho_{K}^{-4} C_{\mathrm{n} \overline{\mathrm{m}} \mathrm{m}} & \text { for } s=-2 \\ \psi_{0} \equiv-C_{\mathrm{lmlm}} & \text { for } s=+2\end{cases}
$$

where an overbar means complex conjugation and $\rho_{K}$ is given in Eq. (5) below. Asymptotically, the leading behavior of the field $\Psi$ represents either the outgoing radiative part of the perturbed Weyl tensor, $(s=-2)$, or the ingoing radiative part, $(s=+2)$.

The components of the Boyer-Lindquist null tetrad for the Kerr background are given by

$$
\begin{aligned}
\left(\mathrm{l}_{\mathrm{K}}^{\alpha}\right) & =\left(\frac{r^{2}+a^{2}}{\Delta}, 1,0, \frac{a}{\Delta}\right), \\
\left(\mathrm{n}_{\mathrm{K}}^{\alpha}\right) & =\frac{1}{2\left(r^{2}+a^{2} \cos ^{2} \theta\right)}\left(r^{2}+a^{2},-\Delta, 0, a\right), \\
\left(\mathrm{m}_{\mathrm{K}}{ }^{\alpha}\right) & =\frac{1}{\sqrt{2}(r+i a \cos \theta)}(i a \sin \theta, 0,1, i / \sin \theta) .
\end{aligned}
$$

One can also define directional derivatives as

$$
\delta=\mathrm{m}_{\mathrm{K}}{ }^{\mu} \partial_{\mu} ; \hat{\Delta}=\mathrm{n}_{\mathrm{K}}{ }^{\mu} \partial_{\mu} ; \hat{D}=\mathrm{l}_{\mathrm{K}}{ }^{\mu} \partial_{\mu}
$$

With the above choice of the tetrad the non-vanishing spin coefficients are (where an overbar stands for complex conjugation)

$$
\begin{aligned}
& \rho_{K}=-\frac{1}{(r-i a \cos \theta)}, \quad \beta_{K}=-\bar{\rho}_{K} \frac{\cot \theta}{2 \sqrt{2}} \\
& \pi_{K}=i a \rho_{K}^{2} \frac{\sin \theta}{\sqrt{2}}, \quad \tau_{K}=-i a \rho_{K} \bar{\rho}_{K} \frac{\sin \theta}{\sqrt{2}} \\
& \mu_{K}=\rho_{K}^{2} \bar{\rho}_{K} \frac{\Delta}{2}, \quad \alpha_{K}=\pi_{K}-\bar{\beta}_{K}, \\
& \gamma=\mu+\rho_{K} \bar{\rho}_{K} \frac{(r-M)}{2},
\end{aligned}
$$

and the only non-vanishing Weyl scalar in the background is

$$
\psi_{2}=M \rho_{K}^{3}
$$

As we mentioned above the Weyl scalars $\psi_{4}$ and $\psi_{0}$ allow a direct computation of the radiation escaping to infinity [8] and going down the horizon. The time-domain formulation is particularly well suited for interfacing with full numerical relativity techniques 4, 1, 5, 2, 3. There are, besides, other physical phenomena of interest such as the self-force on a particle orbiting the hole [16], studies of the horizon structure, and second order perturbations [8], that require the computation of the metric perturbations. 
Starting from the pioneering work of Chzranowski [10] there is a series of papers [15, 27, 24, 21] dealing with the problem of metric reconstruction by the introduction of a potential that satisfies the Teukolsky equation, being neither the $\psi_{4}$ or $\psi_{0}$ describing to the physical situation under study. The problem of relating the potentials introduced to describe the radiation gauges and the physical $\psi_{4}$ and $\psi_{0}$ has been recently studied in Ref. [18]. There, the results are explicitly given for vacuum metric perturbations on the Schwarzschild, i.e. nonrotating, black hole background.

Given the difficulties in obtaining by these method the explicit metric expression for perturbations around a Kerr, i.e. rotating black hole, background, we present here an alternative approach. In this paper we give explicit formulae for the metric reconstruction in the Regge-Wheeler gauge, still for a nonrotating background, but allowing for source terms, bearing in mind, for instance, the applications to the radiation reaction problem. In the next section we will give explicitly the form $\psi_{4}$ and $\psi_{0}$ take in terms of metric perturbation, in the Regge-Wheeler gauge, making explicit use to the multipole decomposition of the metric. In order to invert these expressions, in Sec. 3 we introduce the symmetric and antisymmetric components of the Weyl scalar under the discrete parity transformation. With the help of the field equations of General Relativity in the Regge-Wheeler gauge (reviewed in Appendix A) we succeed in expressing the metric perturbations in terms of $\psi_{4}$ and $\psi_{0}$, including matter terms. In the final section of the paper we describe how to generalize the first few of these steps to the Kerr background case, and speculate about the completion of this program.

\section{Weyl Scalars}

The first step in explicitly constructing the metric perturbations is actually computing the inverse relation, that makes use of the definition of the Weyl scalars (2) in terms of the Weyl tensor. Chrzanowski [10], made this computation explicitly relating the perturbed Weyl scalars to the metric perturbations

$$
\begin{aligned}
\psi_{4}= & \frac{1}{2}\left\{(\bar{\delta}+3 \alpha+\bar{\beta}-\bar{\tau})(\bar{\delta}+2 \alpha+2 \bar{\beta}-\bar{\tau}) h_{\mathrm{nn}}\right. \\
& +(\hat{\triangle}+\bar{\mu}+3 \gamma-\bar{\gamma})(\hat{\triangle}+\bar{\mu}+2 \gamma-2 \bar{\gamma}) h_{\overline{\mathrm{mm}}} \\
& -[(\hat{\triangle}+\bar{\mu}+3 \gamma-\bar{\gamma})(\bar{\delta}-2 \bar{\tau}+2 \alpha) \\
& \left.+(\bar{\delta}+3 \alpha+\bar{\beta}-\bar{\tau})(\hat{\triangle}+2 \bar{\mu}+2 \gamma)] h_{(\mathrm{n} \overline{\mathrm{m}})}\right\},
\end{aligned}
$$

and 


$$
\begin{aligned}
\psi_{0}= & \frac{1}{2}\left\{(\delta-\bar{\alpha}-3 \beta+\bar{\pi})(\delta-2 \bar{\alpha}-2 \beta+\bar{\pi}) h_{\mathrm{ll}}\right. \\
& +(\hat{D}-\bar{\rho}-3 \epsilon+\bar{\epsilon})(\hat{D}-\bar{\rho}-2 \epsilon+2 \bar{\epsilon}) h_{\mathrm{mm}} \\
& -[(\hat{D}-\bar{\rho}-3 \epsilon+\bar{\epsilon})(\delta+2 \bar{\pi}-2 \beta) \\
& \left.+(\delta-\bar{\alpha}-3 \beta+\bar{\pi})(\hat{D}-2 \bar{\rho}-2 \epsilon)] h_{(\mathrm{lm})}\right\},
\end{aligned}
$$

where $h_{\mathrm{nn}}=\mathrm{n}^{\mu} \mathrm{n}^{\nu} h_{\mu \nu}, h_{\mathrm{lm}}=\mathrm{l}^{\mu} \mathrm{m}^{\nu} h_{\mu \nu}$, etc.

Many simplifications are possible in the analysis when the background has spherical symmetry. In the Schwarzschild black hole case expressions (77)-(18) reduce to

$$
\begin{aligned}
\psi_{4}= & \frac{1}{16}\left\{\frac{1}{r^{2}}\left(\partial_{\theta}-\cot \theta-\frac{i}{\sin \theta} \partial_{\varphi}\right)\left(\partial_{\theta}-\frac{i}{\sin \theta} \partial_{\varphi}\right)\right. \\
& \times\left[h_{t t}-2 h_{r t} f+h_{r r} f^{2}\right] \\
& +\left(\partial_{t}-f \partial_{r}+f^{\prime}-\frac{2 f}{r}\right)\left(\partial_{t}-f \partial_{r}\right) \\
& \times \frac{1}{r^{2}}\left[h_{\theta \theta}-\frac{h_{\varphi \varphi}}{\sin ^{2} \theta}-2 i \frac{h_{\theta \varphi}}{\sin \theta}\right] \\
& -\frac{2}{r^{2}}\left(\partial_{t}-f \partial_{r}+f^{\prime}\right)\left(\partial_{\theta}-\cot \theta-\frac{i}{\sin \theta} \partial_{\varphi}\right) \\
& \left.\times\left[h_{t \theta}-i \frac{h_{t \varphi}}{\sin \theta}-f\left(h_{r \theta}-i \frac{h_{r \varphi}}{\sin \theta}\right)\right]\right\}
\end{aligned}
$$

and

$$
\begin{aligned}
\psi_{0}= & \frac{1}{4}\left\{\frac{1}{r^{2}}\left(\partial_{\theta}-\cot \theta+\frac{i}{\sin \theta} \partial_{\varphi}\right)\left(\partial_{\theta}+\frac{i}{\sin \theta} \partial_{\varphi}\right)\right. \\
& \times\left[h_{t t} f^{-2}+2 h_{r t} f^{-1}+h_{r r}\right] \\
& +\left(f^{-1} \partial_{t}+\partial_{r}+\frac{2}{r}\right)\left(f^{-1} \partial_{t}+\partial_{r}\right) \\
& \times \frac{1}{r^{2}}\left[h_{\theta \theta}-\frac{h_{\varphi \varphi}}{\sin ^{2} \theta}+2 i \frac{h_{\theta \varphi}}{\sin \theta}\right] \\
& +\frac{2}{r^{2}}\left(f^{-1} \partial_{t}+\partial_{r}\right)\left(\partial_{\theta}-\cot \theta+\frac{i}{\sin \theta} \partial_{\varphi}\right) \\
& \left.\times\left[f^{-1}\left(h_{t \theta}+i \frac{h_{t \varphi}}{\sin \theta}\right)+h_{r \theta}+i \frac{h_{r \varphi}}{\sin \theta}\right]\right\}
\end{aligned}
$$

where $f=1-2 M / r$ and $f^{\prime}=2 M / r^{2}$.

The imposition of spherical symmetry also carries the following computational advantage: the multipole decomposition of the metric perturbations in terms of spinweighted harmonics ${ }_{-2} Y_{\ell m}(\theta)$ can be performed[22, 19], and even and odd parity 
perturbations decouple so they can be considered independently. Below we shall decompose all metric perturbations in multipoles with index $\ell m$ (not to be confused with the tetrad vectors).

From Eq. (9) and (10) in the Regge-Wheeler gauge $\left(h_{1}^{\ell m}=h_{0}^{\ell m}=G^{\ell m}=0=\right.$ ${ }^{(\text {odd) }} h_{2}^{\ell m}$ ), we get

$$
\begin{aligned}
\psi_{4} \doteq & \sum_{\ell m} \psi_{4}^{\ell m}{ }_{-2} Y_{\ell m}=-\sum_{\ell m} \sqrt{\frac{(\ell-2) !}{(\ell+2) !}} \\
& \times\left\{-\frac{f}{16 r^{2}}\left(H_{0}^{\ell m}-2 H_{1}^{\ell m}+H_{2}^{\ell m}\right)\right. \\
& \left.-\frac{i}{8 r^{2}}\left[\partial_{t}-f \partial_{r}+f^{\prime}\right]\left({ }^{(o d d)} h_{0}^{\ell m}-f{ }^{(o d d)} h_{1}^{\ell m}\right)\right\}{ }_{-2} Y_{\ell m},
\end{aligned}
$$

and

$$
\begin{aligned}
\psi_{0} \doteq & \sum_{\ell m} \psi_{0}^{\ell m}{ }_{+2} Y_{\ell m}=-\sum_{\ell m} \sqrt{\frac{(\ell-2) !}{(\ell+2) !}} \\
& \times\left\{-\frac{1}{4 f r^{2}}\left(H_{0}^{\ell m}+2 H_{1}^{\ell m}+H_{2}^{\ell m}\right)\right. \\
& \left.-\frac{i}{2 f^{2} r^{2}}\left[\partial_{t}+f \partial_{r}-f^{\prime}\right]\left({ }^{(o d d)} h_{0}^{\ell m}+f{ }^{(\text {odd })} h_{1}^{\ell m}\right)\right\}{ }_{+2} Y_{\ell m}
\end{aligned}
$$

These represent our basic equations (real and imaginary parts) that we will use in the next section to express the metric perturbations in terms of $\psi_{4}$ and $\psi_{0}$.

\section{Explicit solution in the Regge-Wheeler gauge}

Two key elements are introduced here in order to complete the inversion of metric coefficients from Eqs. 11]and 12, The first is the decomposition of the Weyl fields into its symmetric and antisymmetric parts with respect to the discrete parity transformation. This allows to separate the even and odd parity perturbations from the multipole decomposed $\psi_{4}, \psi_{0}$, and metric perturbations. For the even parity case this allows to obtain directly two of the four metric coefficients. In order to obtain the other two, we have to resource to the General Relativity field equations, which represent the other key element in the inversion process. For the odd parity case, one ends up with first order differential relations that can be brought to explicit integrals (previous simplification by making us of the odd parity field equations).

\subsection{Even parity}

Let us define the symmetric and antisymmetric Weyl scalar fields as [18]

$$
\psi^{ \pm}=\frac{1}{2}\left[\psi^{\ell, m} \pm(-)^{m} \bar{\psi}^{\ell,-m}\right]
$$


where for notational simplicity we dropped the $\ell, m$ indexes. Thus, given the symmetric nature of the even parity metric perturbations, Eqs. (11) and (12) take the form

$$
\psi_{4}^{+}=\frac{f}{16 r^{2}} \sqrt{\frac{(\ell-2) !}{(\ell+2) !}}\left(H_{0}^{\ell m}-2 H_{1}^{\ell m}+H_{2}^{\ell m}\right),
$$

and

$$
\psi_{0}^{+}=\frac{1}{4 f r^{2}} \sqrt{\frac{(\ell-2) !}{(\ell+2) !}}\left(H_{0}^{\ell m}+2 H_{1}^{\ell m}+H_{2}^{\ell m}\right) .
$$

From Eqs. (14) and (15) we can obtain the $\ell m$ components of the metric perturbations as follows

$$
H_{1}^{\ell m}(r, t)=-\frac{4 r^{2}}{f} \sqrt{\frac{(\ell+2) !}{(\ell-2) !}}\left[\psi_{4}^{+}-\frac{f^{2}}{4} \psi_{0}^{+}\right]
$$

and

$$
H_{0}^{\ell m}(r, t)+H_{2}^{\ell m}(r, t)=\frac{8 r^{2}}{f} \sqrt{\frac{(\ell+2) !}{(\ell-2) !}}\left[\psi_{4}^{+}+\frac{f^{2}}{4} \psi_{0}^{+}\right]
$$

We now bring into the play the Hilbert-Einstein's equations in the Regge-Wheeler gauge, Eq. (A.14) give,

$$
H_{0}^{\ell m}(r, t)-H_{2}^{\ell m}(r, t)=\frac{16 \pi r^{2}}{\sqrt{2 \lambda(\lambda+1)}} F^{\ell m},
$$

where $F_{\ell m}$ is a source term given in Table III of Ref. 29] (See also Appendix B.) This allows to find the metric perturbations for $H_{0}^{\ell m}, H_{1}^{\ell m}$, and $H_{2}^{\ell m}$. The last metric coefficient has to be found by use of the Hilbert-Einstein equations $\left[K^{\ell m}\right.$ and $H_{0}^{\ell m}-H_{2}^{\ell m}$ give a measure of the trace of the even parity sector in the Regge-Wheeler gauge, so it does not appear in the Weyl scalars since the Weyl tensor is traceless.]

Using Eq. (A.12) we can solve for $\partial_{r} K^{\ell m}$ and then replace it in Eq. (A.8) to find $K^{\ell m}$ in terms of the other even parity metric coefficients and source terms

$$
\begin{aligned}
& K(r, t)^{\ell m}= \\
& 2 \frac{(r-M) \frac{\partial}{\partial r} H_{0}^{\ell m}(r, t)}{\lambda}+\frac{(r-2 M) r \frac{\partial^{2}}{\partial r^{2}} H_{0}^{\ell m}(r, t)}{\lambda} \\
& -\frac{r^{2} \frac{\partial^{2}}{\partial r \partial t} H_{1}^{\ell m}(r, t)}{\lambda}+\frac{M \frac{\partial}{\partial r} H_{2}^{\ell m}(r, t)}{\lambda} \\
& -\frac{\left(2 r^{2}-8 r M+9 M^{2}\right) H_{0}^{\ell m}(r, t)}{r \lambda(r-2 M)} \\
& +\frac{\left(-r^{2} \lambda+2 r M \lambda+3 M^{2}-2 r M\right) H_{2}^{\ell m}(r, t)}{r \lambda(r-2 M)} \\
& +\frac{r(-3 r+7 M) \frac{\partial}{\partial t} H_{1}(r, t)}{\lambda(r-2 M)}+8 \frac{(r-2 M) \pi r^{2} \frac{\partial}{\partial r} B^{\ell m}(r, t)}{\sqrt{\lambda+1} \lambda} \\
& -8 \frac{(7 M-4 r) \pi r B^{\ell m}(r, t)}{\sqrt{\lambda+1} \lambda}-\frac{A_{0}^{\ell m}(r, t) r^{3}}{\lambda(r-2 M)} .
\end{aligned}
$$


This form of the metric coefficient $K^{\ell m}$, involves second derivatives of the Weyl scalars. One can consider an alternative integral form (on the hypersurface $t=$ constant) derived from Eq. (A.12)

$$
\begin{aligned}
K^{\ell m}= & H_{0}^{\ell m}+\int_{2 M}^{r} \frac{d r}{1-\frac{2 M}{r}}\left[-\frac{\partial H_{1}^{\ell m}}{\partial t}+\frac{2 M}{r^{2}} H_{0}^{\ell m}\right. \\
& \left.-16 \pi(r-2 M) \frac{F^{\ell m}}{\sqrt{2 \lambda(\lambda+1)}}-\frac{8 \pi(r-2 M)}{\sqrt{\lambda+1}} B^{\ell m}\right]
\end{aligned}
$$

It is worth mentioning here that if the source is modeled as a particle (represented by a Dirac's Delta) the above metric coefficients are continuous $\left(C^{0}\right)$ for headon collisions 16] at the location of the particle. However, for more general orbits they do not all appear to be continuous but some of them behave as a Dirac's Delta. For instance, one can see that from expression (18).$F^{\ell m}$ is proportional to a Dirac's Delta, as given in Table A1 hence, at least $H_{2}^{\ell m}$ or $H_{2}^{\ell m}$ have to behave as $\delta[r-R(t)]$.

\subsection{Odd parity}

From Eq. (11) and (12), given the antisymmetric behaviour of the odd parity metric coefficients, we get

$$
\psi_{4}^{-}=\frac{i}{8 r^{2}} \sqrt{\frac{(\ell-2) !}{(\ell+2) !}}\left[\partial_{t}-f \partial_{r}+f^{\prime}\right]\left({ }^{(o d d)} h_{0}^{\ell m}-f^{(\text {odd })} h_{1}^{\ell m}\right)
$$

and

$$
\psi_{0}^{-}=\frac{i}{2 f^{2} r^{2}} \sqrt{\frac{(\ell-2) !}{(\ell+2) !}}\left[\partial_{t}+f \partial_{r}-f^{\prime}\right]\left({ }^{(o d d)} h_{0}^{\ell m}+f^{(o d d)} h_{1}^{\ell m}\right) .
$$

A linear combination of these previous equations produces

$$
\psi_{4}^{-}+\frac{f^{2}}{4} \psi_{0}^{-}=\frac{i}{4 r^{2}} \sqrt{\frac{(\ell-2) !}{(\ell+2) !}}\left[\partial_{t}^{(\text {odd })} h_{0}^{\ell m}+\left(f \partial_{r}-f^{\prime}\right)\left(f^{(\text {odd })} h_{1}^{\ell m}\right)\right]
$$

and

$$
\psi_{4}^{-}-\frac{f^{2}}{4} \psi_{0}^{-}=\frac{-i}{4 r^{2}} \sqrt{\frac{(\ell-2) !}{(\ell+2) !}}\left[f \partial_{t}^{\left({ }^{(o d d)}\right.} h_{1}^{\ell m}+\left(f \partial_{r}-f^{\prime}\right)\left({ }^{(o d d)} h_{0}^{\ell m}\right)\right] .
$$

From Eq. (A.17) we can substitute $\partial_{t} h_{0}^{\ell m}$ into Eq. (23) leading to the equation

$$
\begin{aligned}
& \partial_{r} h_{1}^{\ell m}+\left(\frac{f^{\prime}}{2 f}\right) h_{1}^{\ell m}=S_{1}^{\ell m}(r, t) \doteq \\
& \frac{-2 i r^{2}}{f^{2}} \sqrt{\frac{(\ell+2) !}{(\ell-2) !}}\left(\psi_{4}^{-}+\frac{f^{2}}{4} \psi_{0}^{-}\right)-\frac{2 \pi i r^{2} D_{\ell m}}{f \sqrt{\lambda(\lambda+1)}}
\end{aligned}
$$


which integrated produces

$$
h_{1}^{\ell m}=\frac{1}{\sqrt{1-\frac{2 M}{r}}}\left\{\int_{2 M}^{r} S_{1}^{\ell m}\left(r^{\prime}, t\right) \sqrt{1-\frac{2 M}{r^{\prime}}} d r^{\prime}+C_{1}^{\ell m}(t)\right\},
$$

where $C_{1}^{\ell m}$ is an integration constant, that in vacuum and with vanishing $\psi_{0}$ and $\psi_{4}$ can be taken to vanish [26].

Knowing now the form of $h_{1}^{\ell m}$ we can use Eq (24) to find a differential equation for $h_{0}^{\ell m}$

$$
\partial_{r} h_{0}^{\ell m}-\left(\frac{f^{\prime}}{f}\right) h_{0}^{\ell m}=S_{0}^{\ell m}(r, t) \doteq \frac{4 i r^{2}}{f} \sqrt{\frac{(\ell+2) !}{(\ell-2) !}}\left(\psi_{4}^{-}-\frac{f^{2}}{4} \psi_{0}^{-}\right)-\partial_{t} h_{1}^{\ell m},
$$

with solution

$$
h_{0}^{\ell m}=\left(1-\frac{2 M}{r}\right)\left\{\int_{2 M}^{r} \frac{S_{0}^{\ell m}\left(r^{\prime}, t\right)}{1-\frac{2 M}{r^{\prime}}} d r^{\prime}+C_{0}^{\ell m}(t)\right\} .
$$

Again, $C_{0}^{\ell m}$ is an integration constant, that in vacuum and with vanishing $\psi_{0}$ and $\psi_{4}$ can be taken to vanish.

This essentially completes the work of expressing the metric perturbations in terms of the computed $\psi_{0}$ and $\psi_{4}$ expressed in the time domain. For the even parity case it contains second derivatives of the Weyl scalars (unlike the corresponding expressions for the radiation gauge [18.) For the odd parity case, solutions (29) and (26) are written in an integral form.

A last observation applies here, since the spin weight of the Weyl scalars are $s= \pm 2$ they do not contain multipole modes $\ell=0$ and $\ell=1$, hence we need to give them by directly solving the field equations for the metric coefficients. The Regge-Wheeler gauge do not completely allow to determine them, because there are one extra degree of freedom for $\ell=1$ and two degrees of freedom for $\ell=0$. Zerilli [29] has made choices to fix this extra freedom that allowed him to solve analytically for the metric coefficients. In Ref. [6] a different choice was made to make those coefficients continuous in the headon collision of extreme mass black holes. Finally in Ref. 12] the metric coefficients for $\ell=0,1$ have been found in the harmonic gauge, for particles in circular orbits.

\section{Discussion of Kerr perturbations}

A possible generalization of the Regge-Wheeler gauge conditions for spherically symmetric backgrounds, but where perturbations are not decomposed into multipoles 
is [7]

$$
\begin{aligned}
(\sin \theta)^{2} h_{\theta \theta}-h_{\phi \phi} & =0, \\
h_{\theta \phi} & =0, \\
\sin \theta \partial_{\theta}\left(\sin \theta h_{t \theta}\right)+\partial_{\phi} h_{t \phi} & =0, \\
\sin \theta \partial_{\theta}\left(\sin \theta h_{r \theta}\right)+\partial_{\phi} h_{r \phi} & =0 .
\end{aligned}
$$

The first equation above leads to the condition $G^{\ell m}=0$. The second gives then ${ }^{(o d d)} h_{2}^{\ell m}=0$. The other two differential conditions are chosen such that they lead to ${ }^{(\text {even })} h_{0}^{\ell m}=0={ }^{(\text {even })} h_{1}^{\ell m}$, but allow ${ }^{(\text {odd })} h_{0}^{\ell m} \neq 0$ and ${ }^{(\text {odd })} h_{1}^{\ell m} \neq 0$ be unconstrained.

Now we will consider the generalization of the Regge-Wheeler gauge in the NewmanPenrose formalism. In this formalism, the first two Regge-Wheeler conditions, Eqs. (31) and (32), have a simple generalization

$$
h_{\mathrm{mm}}=\mathrm{m}^{\mu} \mathrm{m}^{\nu} h_{\mu \nu}=0 .
$$

Note that requiring that the real and imaginary parts vanish contains both conditions. Obviously,

$$
h_{\overline{\mathrm{mm}}}=\overline{\mathrm{m}}^{\mu} \overline{\mathrm{m}}^{\nu} h_{\mu \nu}=0,
$$

also holds. Note that conditions (35) and (36) are invariant under type III (spin-boosts) transformations of the background tetrad

$$
\mathrm{l} \rightarrow A^{2} \mathrm{l}, \quad \mathrm{n} \rightarrow A^{-2} \mathrm{n}, \quad \mathrm{m} \rightarrow e^{2 i \Theta} \mathrm{m}, \quad \overline{\mathrm{m}} \rightarrow e^{-2 i \Theta} \overline{\mathrm{m}} .
$$

This is an important feature, since the Kinnersley choice of the tetrad, with the spin coefficient $\epsilon=0$ is just a simple, but arbitrary way of fixing the spin-boost freedom. In contrast the convenient choice of the $l$ and $n$ tetrad vectors along the repeated principal null directions of the Kerr background allows to single out wave equations for the perturbations of $\psi_{4}$ and $\psi_{0}$.

To generalize the differential conditions (33) and (34) one can resort to the type III transformation properties of the $\delta$ and $\bar{\delta}$, as well as spin coefficient operators in the Kerr background acting on the metric coefficients $h_{(\mathrm{lm})}$ and $h_{(\mathrm{n} \overline{\mathrm{m}})}$. The objects

$$
(\delta-2 \bar{\alpha}) h_{(\mathrm{l} \overline{\mathrm{m}})} \rightarrow A^{2}(\delta-2 \bar{\alpha}) h_{(\mathrm{l} \overline{\mathrm{m}})},
$$

and

$$
(\bar{\delta}+2 \bar{\beta}) h_{(\mathrm{nm})} \rightarrow A^{-2}(\bar{\delta}+2 \bar{\beta}) h_{(\mathrm{nm})},
$$

transform as objects of spin- 0 and boost weight +1 and -1 respectively under type III transformations of the background tetrad (37).

In order to reproduce the differential conditions (33) and (34) in the Schwarzschild limit, one can then require

$$
\Re\left[(\delta-2 \bar{\alpha}+a \tau-b \bar{\pi}) h_{(\mathrm{l} \overline{\mathrm{m}})}\right]=0,
$$

and

$$
\Re\left[(\bar{\delta}+2 \bar{\beta}+c \bar{\tau}-d \pi) h_{(\mathrm{nm})}\right]=0,
$$


Where $\Re$ is the real part and where following [28 we introduced additional terms containing spin coefficients with spin \pm 1 respectively and boost 0 multiplied by constants $a, b, c, d$ to allow for a more general choice of the gauge. These constants can be readily chosen to facilitate the metric reconstruction or, in other contexts, to impose further symmetries or facilitate the numerical integration of General Relativity field equations, etc. It also stresses the ambiguities in generalizing the Regge-Wheeler gauge on the Kerr background.

Note also that conditions (40) and (41) are invariant under type III transformations as well. A crucial role in achieving that was played by the spin- 0 transformation properties of the constructed object, allowing invariance of its Real part.

Independently, we can try to proceed along the lines of the previous sections with now a simple mode decomposition of the metric coefficients. For instance

$$
h_{(\mathrm{lm})}^{ \pm}=\frac{1}{2}\left[h_{(\mathrm{lm})}^{m} \pm(-)^{m} h_{(\mathrm{l} \overline{\mathrm{m}})}^{-m}\right]
$$

where we decomposed

$$
h_{(\operatorname{lm})}(t, r, \theta, \varphi)=\sum_{m} e^{i m \varphi} h_{(\operatorname{lm})}^{m}(t, r, \theta)
$$

We can now replace this decomposition directly into Eqs. (17) and (8) for $\psi_{4}$ and $\psi_{0}$ or the following more convenient form making use of Ref. [25], Eq. (2.11), where we have the following identity

$$
\begin{aligned}
& {[\hat{D}-(p+1) \epsilon+\bar{\epsilon}+q \rho-\bar{\rho}](\delta-p \beta+q \tau)} \\
& =[\delta-(p+1) \beta-\bar{\alpha}+q \tau+\bar{\pi}](\hat{D}-p \epsilon+q \rho),
\end{aligned}
$$

and the identity derived from it exchanging tetrads $\mathrm{l} \rightarrow \mathrm{n}$ and $\mathrm{m} \rightarrow \overline{\mathrm{m}}$.

$$
\begin{aligned}
& {[\hat{\Delta}+(p+1) \gamma-\bar{\gamma}-q \mu+\bar{\mu}](\bar{\delta}+p \alpha-q \pi)} \\
& =[\bar{\delta}+(p+1) \alpha+\bar{\beta}-q \pi-\bar{\tau}](\hat{\Delta}+p \gamma-q \mu) .
\end{aligned}
$$

Using $p=2$ and $q=0$ in the above identities allows us to rewrite Eqs. (7) and (8) as

$$
\begin{aligned}
\psi_{4}= & \frac{1}{2}\left\{(\bar{\delta}+3 \alpha+\bar{\beta}-\bar{\tau})(\bar{\delta}+2 \alpha+2 \bar{\beta}-\bar{\tau}) h_{\mathrm{nn}}\right. \\
& +(\hat{\triangle}+\bar{\mu}+3 \gamma-\bar{\gamma})(\hat{\triangle}+\bar{\mu}+2 \gamma-2 \bar{\gamma}) h_{\overline{\mathrm{mm}}} \\
& -[2(\hat{\triangle}+\bar{\mu}+3 \gamma-\bar{\gamma})(\bar{\delta}-\bar{\tau}+2 \alpha) \\
& \left.+2(\bar{\delta}+3 \alpha+\bar{\beta}-\bar{\tau})(\bar{\mu})] h_{(\mathrm{n} \overline{\mathrm{m}})}\right\},
\end{aligned}
$$


and

$$
\begin{aligned}
\psi_{0}= & \frac{1}{2}\left\{(\delta-\bar{\alpha}-3 \beta+\bar{\pi})(\delta-2 \bar{\alpha}-2 \beta+\bar{\pi}) h_{\mathrm{ll}}\right. \\
& +(\hat{D}-\bar{\rho}-3 \epsilon+\bar{\epsilon})(\hat{D}-\bar{\rho}-2 \epsilon+2 \bar{\epsilon}) h_{\mathrm{mm}} \\
& -[2(\hat{D}-\bar{\rho}-3 \epsilon+\bar{\epsilon})(\delta+\bar{\pi}-2 \beta) \\
& \left.-2(\delta-\bar{\alpha}-3 \beta+\bar{\pi})(\bar{\rho})] h_{(\mathrm{lm})}\right\}
\end{aligned}
$$

A choice of the symmetric tetrad Appendix D will further simplify the appearance of the equations. At this point we impose our gauge condition on $h_{(\operatorname{lm})}^{+}$and $h_{(\mathrm{n} \overline{\mathrm{m}})}^{+}$. Then, paralleling the work done in the nonrotating limit, we could make further progress by writing explicitly the Newman-Penrose equations in terms of metric perturbations, for finally using these equations to obtain decoupled expressions for some metric coefficients. The completion of this program remains an open issue and goes beyond the scope of this paper. We leave this for future research.

\section{Acknowledgments}

The author specially thanks L. Barack, L. Price and B. Whiting for useful discussions during the development of this manuscript. C.O.L. gratefully acknowledges the support of the NASA Center for Gravitational Wave Astronomy at The University of Texas at Brownsville (NAG5-13396), and from NSF grants PHY-0140326 and PHY-0354867.

\section{Appendix A. Hilbert-Einstein equations in the Regge-Wheeler gauge}

Because of some misprints in the original Zerilli's paper 29] we reproduce here the relevant equations for our discussions (See also Ref. [13, 23]).

The metric perturbations on a Schwarzschild background

$$
d s^{2}=-\left(1-\frac{2 M}{r}\right) d t^{2}+\left(1-\frac{2 M}{r}\right)^{-1} d r^{2}+r^{2}\left(d \theta^{2}+\sin \theta d \varphi^{2}\right),
$$

can be decomposed into spherical harmonics 29

$$
h_{\mu \nu}^{\text {even }}=\left|\begin{array}{cccc}
\left(1-\frac{2 M}{r}\right) H_{0}^{\ell m}(t, r) & H_{1}^{\ell m}(t, r) & h_{0}^{\ell m}(t, r) \frac{\partial}{\partial \theta} & h_{0}^{\ell m}(t, r) \frac{\partial}{\partial \varphi} \\
H_{1}^{\ell m}(t, r) & \frac{H_{2}^{\ell m}(t, r)}{\left(1-\frac{2 M}{r}\right)} & h_{1}^{\ell m}(t, r) \frac{\partial}{\partial \theta} & h_{1}^{\ell m}(t, r) \frac{\partial}{\partial \varphi} \\
h_{0}^{\ell m}(t, r) \frac{\partial}{\partial \theta} & h_{1}^{\ell m}(t, r) \frac{\partial}{\partial \theta} & r^{2}\left[K^{\ell m}+G^{\ell m} \frac{\partial^{2}}{\partial \theta^{2}}\right] & r^{2} G^{\ell m}(t, r) \frac{\hat{X}_{\ell m}}{2} \\
h_{0}^{\ell m}(t, r) \frac{\partial}{\partial \varphi} & h_{1}^{\ell m}(t, r) \frac{\partial}{\partial \varphi} & r^{2} G^{\ell m}(t, r) \frac{\hat{X}_{\ell m}}{2} & r^{2}\left[K^{\ell m} \sin ^{2} \theta+G^{\ell m} \hat{Z}_{\ell m}\right]
\end{array}\right| Y_{\ell m}(\theta, \varphi)
$$

for the even parity modes. 
Reconstruction of Black Hole Metric Perturbations from Weyl Curvature II

And

$$
h_{\mu \nu}^{\text {odd }}=\left|\begin{array}{cccc}
0 & 0 & -h_{0}^{\ell m}(t, r) \frac{\partial}{\sin \theta \partial \varphi} & h_{0}^{\ell m}(t, r) \frac{\sin \theta \partial}{\partial \theta} \\
0 & 0 & -h_{1}^{\ell m}(t, r) \frac{\partial}{\sin \theta \partial \varphi} & h_{1}^{\ell m}(t, r) \frac{\sin \theta \partial}{\partial \theta} \\
-h_{0}^{\ell m}(t, r) \frac{\partial}{\sin \theta \partial \varphi} & -h_{1}^{\ell m}(t, r) \frac{\partial}{\sin \theta \partial \varphi} & h_{2}^{\ell m}(t, r) \frac{\hat{X}_{\ell m}}{2 \sin \theta} & h_{2}^{\ell m}(t, r) \sin \theta \frac{\hat{W}_{\ell m}}{2} \\
h_{0}^{\ell m}(t, r) \frac{\sin \theta \partial}{\partial \theta} & h_{1}^{\ell m}(t, r) \frac{\sin \theta \partial}{\partial \theta} & h_{2}^{\ell m}(t, r) \sin \theta \frac{\hat{W}_{\ell m}}{2} & -h_{2}^{\ell m}(t, r) \sin \theta \frac{\hat{X}_{\ell m}}{2}
\end{array}\right| Y_{\ell m}(\theta, \varphi)
$$

for the odd parity modes. Where $g_{\mu \nu}=g_{\mu \nu}^{\mathrm{Schw}}+h_{\mu \nu}$.

Above we used Zerilli's notation

$$
\begin{gathered}
\hat{X}_{\ell m} \doteq 2 \frac{\partial}{\partial \varphi}\left(\frac{\partial}{\partial \theta}-\cot \theta\right) \\
\hat{W}_{\ell m} \doteq\left(\frac{\partial^{2}}{\partial \theta^{2}}-\cot \theta \frac{\partial}{\partial \theta}-\frac{1}{\sin ^{2} \theta} \frac{\partial^{2}}{\partial \varphi^{2}}\right),
\end{gathered}
$$

and

$$
\hat{Z}_{\ell m} \doteq\left(\frac{\partial^{2}}{\partial \varphi^{2}}+\sin \theta \cos \theta \frac{\partial}{\partial \theta}\right) .
$$

We will also introduce Zerilli's $\lambda$

$$
\lambda=(\ell-1)(\ell+2) / 2 .
$$

Appendix A.1. Even Parity

The Zerilli's (C7a)-(C7g) equations with corrections are

$$
\begin{aligned}
& \left(1-\frac{2 M}{r}\right)^{2} \frac{\partial^{2} K^{\ell m}}{\partial r^{2}}+\frac{1}{r}\left(1-\frac{2 M}{r}\right)\left(3-\frac{5 M}{r}\right) \frac{\partial K^{\ell m}}{\partial r} \\
& -\frac{1}{r}\left(1-\frac{2 M}{r}\right)^{2} \frac{\partial H_{2}^{\ell m}}{\partial r}-\frac{1}{r^{2}}\left(1-\frac{2 M}{r}\right)\left(H_{2}^{\ell m}-K^{\ell m}\right) \\
& -\frac{(\lambda+1)}{r^{2}}\left(1-\frac{2 M}{r}\right)\left(H_{2}^{\ell m}+K^{\ell m}\right)=-8 \pi A_{\ell m}^{(0)}, \\
& \frac{\partial}{\partial t}\left[\frac{\partial K^{\ell m}}{\partial r}+\frac{1}{r}\left(K^{\ell m}-H_{2}^{\ell m}\right)-\frac{M}{r(r-2 M)} K^{\ell m}\right]-\frac{(\lambda+1)}{r^{2}} H_{1}^{\ell m} \\
& =-4 \sqrt{2} \pi i A_{\ell m}^{(1)}, \\
& \left(\frac{r}{r-2 M}\right)^{2} \frac{\partial^{2} K^{\ell m}}{\partial t^{2}}-\frac{r-M}{r(r-2 M)} \frac{\partial K^{\ell m}}{\partial r}-\frac{2}{r-2 M} \frac{\partial H_{1}^{\ell m}}{\partial t} \\
& +\frac{1}{r} \frac{\partial H_{0}^{\ell m}}{\partial r}+\frac{1}{r(r-2 M)}\left(H_{2}^{\ell m}-K^{\ell m}\right) \\
& +\frac{(\lambda+1)}{r(r-2 M)}\left(K^{\ell m}-H_{0}^{\ell m}\right)=-8 \pi A_{\ell m},
\end{aligned}
$$


Reconstruction of Black Hole Metric Perturbations from Weyl Curvature II

$$
\begin{aligned}
& \frac{\partial}{\partial r}\left[\left(1-\frac{2 M}{r}\right) H_{1}^{\ell m}\right]-\frac{\partial}{\partial t}\left(H_{2}^{\ell m}+K^{\ell m}\right)=\frac{8 \pi i r}{\sqrt{\lambda+1}} B_{\ell m}^{(0)}, \\
& -\frac{\partial H_{1}^{\ell m}}{\partial t}+\left(1-\frac{2 M}{r}\right) \frac{\partial}{\partial r}\left(H_{0}^{\ell m}-K^{\ell m}\right)+\frac{2 M}{r^{2}} H_{0}^{\ell m} \\
& +\frac{1}{r}\left(1-\frac{M}{r}\right)\left(H_{2}^{\ell m}-H_{0}^{\ell m}\right)=\frac{8 \pi(r-2 M)}{\sqrt{\lambda+1}} B_{\ell m}, \\
& -\frac{r}{r-2 M} \frac{\partial^{2} K^{\ell m}}{\partial t^{2}}+\left(1-\frac{2 M}{r}\right) \frac{\partial^{2} K^{\ell m}}{\partial r^{2}}+\frac{2}{r}\left(1-\frac{M}{r}\right) \frac{\partial K^{\ell m}}{\partial r} \\
& -\frac{r}{r-2 M} \frac{\partial^{2} H_{2}^{\ell m}}{\partial t^{2}}+2 \frac{\partial^{2} H_{1}^{\ell m}}{\partial t \partial r}-\left(1-\frac{2 M}{r}\right) \frac{\partial^{2} H_{0}^{\ell m}}{\partial r^{2}} \\
& +\frac{2(r-M)}{r(r-2 M)} \frac{\partial H_{1}^{\ell m}}{\partial t}-\frac{1}{r}\left(1-\frac{M}{r}\right) \frac{\partial H_{2}^{\ell m}}{\partial r}-\frac{r+M}{r^{2}} \frac{\partial H_{0}^{\ell m}}{\partial r} \\
& +\frac{(\lambda+1)}{r^{2}}\left(H_{0}^{\ell m}-H_{2}^{\ell m}\right)=8 \sqrt{2} \pi G_{\ell m}^{(s)}, \\
& \frac{H_{0}^{\ell m}-H_{2}^{\ell m}}{2}=\frac{8 \pi r^{2} F_{\ell m}}{\sqrt{2 \lambda(\lambda+1)}} \cdot
\end{aligned}
$$

\section{Appendix A.2. Odd Parity}

The Einstein equation in the Regge-Wheeler gauge for the odd parity sector are [See Zerilli's 30] equations (C6a)-(C6c)). Note the corrections to the source terms.]

$$
\begin{aligned}
& \frac{\partial^{2} h_{0}^{\ell m}}{\partial r^{2}}-\frac{\partial^{2} h_{1}^{\ell m}}{\partial r \partial t}-\frac{2}{r} \frac{\partial h_{1}^{\ell m}}{\partial t}+\left[\frac{4 M}{r^{2}}-\frac{2(\lambda+1)}{r}\right] \frac{h_{0}^{\ell m}}{r-2 M} \\
&=-\frac{8 \pi r Q_{\ell m}^{(0)}}{\left(1-\frac{2 M}{r}\right) \sqrt{(\lambda+1)}}, \\
& \frac{\partial^{2} h_{1}^{\ell m}}{\partial t^{2}}-\frac{\partial^{2} h_{0}^{\ell m}}{\partial r \partial t}+\frac{2}{r} \frac{\partial h_{0}^{\ell m}}{\partial t}+2 \lambda(r-2 M) \frac{h_{1}^{\ell m}}{r^{3}}=\frac{8 \pi i(r-2 M) Q_{\ell m}}{\sqrt{(\lambda+1)}}, \\
&\left(1-\frac{2 M}{r}\right) \frac{\partial h_{1}^{\ell m}}{\partial r}-\frac{1}{\left(1-\frac{2 M}{r}\right)} \frac{\partial h_{0}^{\ell m}}{\partial t}+\frac{2 M}{r^{2}} h_{1}^{\ell m}=-\frac{4 \pi i r^{2} D_{\ell m}}{\sqrt{\lambda(\lambda+1)}},
\end{aligned}
$$

where $Q_{\ell m}^{(0)}, Q_{\ell m}$ and $D_{\ell m}$ give the multipole decomposition of the energy-momentum tensor (See Table A1).

\section{Appendix A.3. Source terms}

Table A1 gives the source terms produced by an orbiting particle in the Schwarzschild background after decomposition of the Stress-Energy Tensor into tensor harmonics. There, $U^{0}(t)=d t / d \tau$, is the zeroth component of the four-velocity of the particle and $\Omega_{p}(t)$ is its angular location.

Table A1. Energy-momentum-Stress Tensor in terms of Tensor Harmonics 


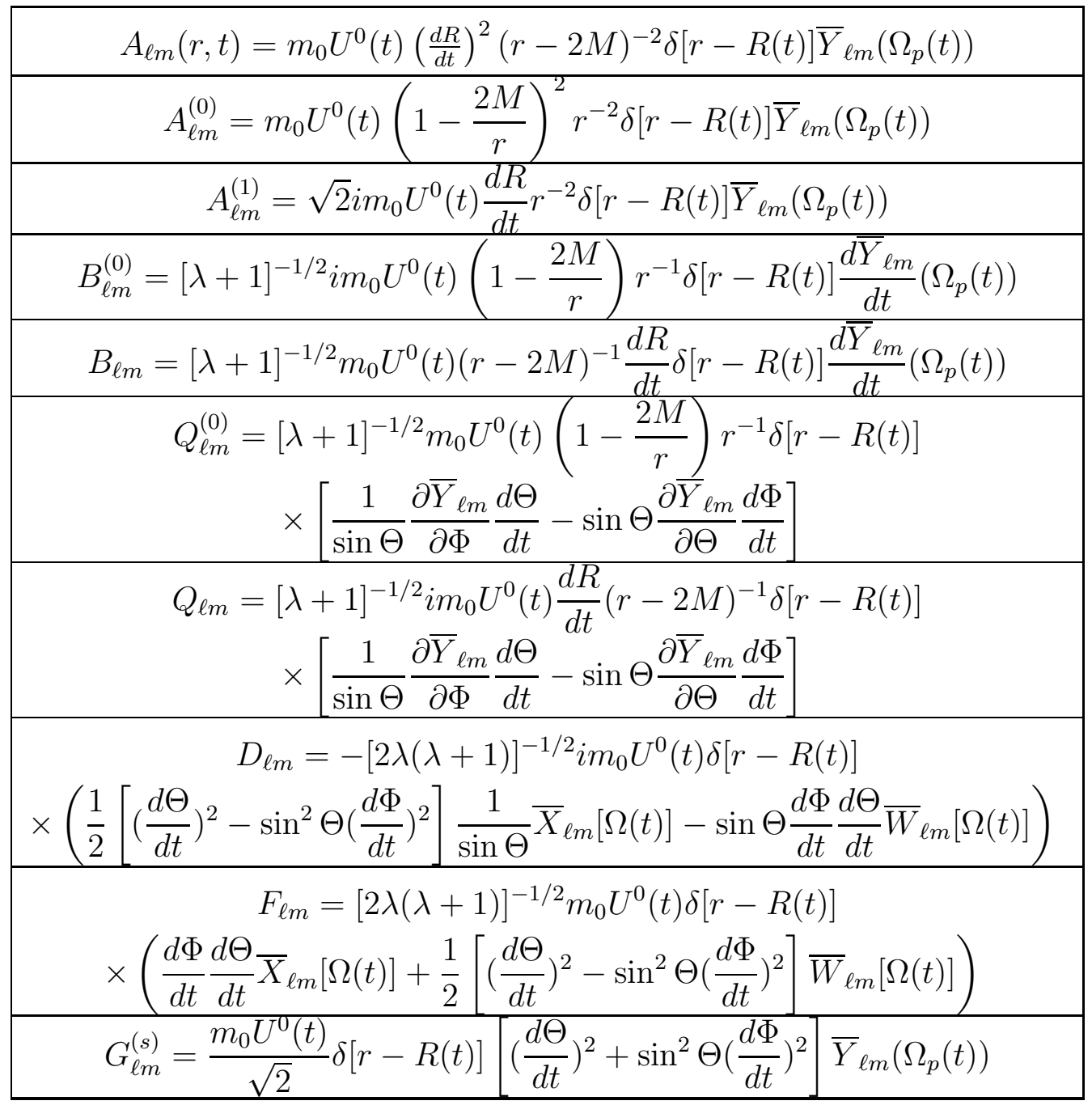

\section{Appendix B. Reconstruction in terms of metric perturbations waveforms}

Here we recall the metric reconstruction in the original Schwarzschild perturbations approach based on waveforms for the even and odd parity perturbations (Zerilli's and Regge-Wheeler respectively). We first introduce the gauge invariant expressions for these waveforms. We then make use of the general relativistic field equations, in the Regge-Wheeler gauge, to solve for the metric perturbations, including nonvanishing matter terms.

\section{Appendix B.1. Even Parity}

We consider the following waveform[17] in terms of generic metric perturbations in the Regge-Wheeler notation

$$
\begin{aligned}
\psi_{\text {even }}^{\ell m}(r, t) & =\frac{r}{(\lambda+1)}\left[K^{\ell m}+\frac{r-2 M}{\lambda r+3 M}\left(H_{2}^{\ell m}-r \partial_{r} K^{\ell m}\right)\right] \\
& +\frac{r-2 M}{\lambda r+3 M}\left[r^{2} \partial_{r} G^{\ell m}-2 h_{1}^{\ell m}\right],
\end{aligned}
$$


Reconstruction of Black Hole Metric Perturbations from Weyl Curvature II

This is related to Zerilli's [30] even parity waveforms $\psi_{Z e r}^{\ell m}$ by

$$
\partial_{t} \psi_{\mathrm{even}}^{\ell m}=\psi_{Z e r}^{\ell m}-\frac{4 \pi i \sqrt{2} r^{2}(r-2 M) A_{\ell m}^{(1)}}{(\lambda+1)(\lambda r+3 M)},
$$

where for an orbiting particle 30 .

$$
A_{\ell m}^{(1)}=i m_{0} \sqrt{2}\left(\frac{U^{0}(t)}{r^{2}}\right)\left(\frac{d R}{d t}\right) \bar{Y}_{\ell m} \delta[r-R(t)],
$$

and it relates to Moncrief's[19] waveform $\psi_{M o n}^{\ell m}$ by

$$
\psi_{\mathrm{even}}^{\ell m}=\frac{\psi_{M o n}^{\ell m}}{(\lambda+1)} .
$$

The $t t$ component of Hilbert-Einstein's equations gives us the Hamiltonian constraint. In the Regge-Wheeler gauge $\left(h_{1}^{\ell m}=h_{0}^{\ell m}=G^{\ell m}=0\right)$ it is given by Eq. (A.8). Only two metric coefficients $\left(K^{\ell m}\right.$ and $\left.H_{2}^{\ell m}\right)$ appear in this equation and none of its time derivatives. Considering the Regge-Wheeler gauge, the definition of $\psi_{\text {even }}^{\ell m}$ (see Eq. (B.1), and the Hamiltonian constraint, Eq. (A.8), we can express these two metric coefficients in terms of $\psi_{\text {even }}^{\ell m}$ (and source terms) only

$$
\begin{aligned}
K^{\ell m}= & \frac{6 M^{2}+3 M \lambda r+\lambda(\lambda+1) r^{2}}{r^{2}(\lambda r+3 M)} \psi_{\mathrm{even}}^{\ell m}+\left(1-\frac{2 M}{r}\right) \partial_{r} \psi_{\mathrm{even}}^{\ell m} \\
& -\frac{8 \pi r^{3} A_{\ell m}^{(0)}}{(\lambda+1)(\lambda r+3 M)},
\end{aligned}
$$

and

$$
\begin{aligned}
& H_{2}^{\ell m}=-\frac{9 M^{3}+9 \lambda M^{2} r+3 \lambda^{2} M r^{2}+\lambda^{2}(\lambda+1) r^{3}}{r^{2}(\lambda r+3 M)^{2}} \psi_{\text {even }}^{\ell m} \\
& +\frac{3 M^{2}-\lambda M r+\lambda r^{2}}{r(\lambda r+3 M)} \partial_{r} \psi_{\text {even }}^{\ell m}+(r-2 M) \partial_{r}^{2} \psi_{\text {even }}^{\ell m} \\
& -\frac{8 \pi r^{4}}{(\lambda+1)(\lambda r+3 M)} \partial_{r} A_{\ell m}^{(0)} \\
& +\frac{8 \pi r^{3}\left(\lambda^{2} r^{2}-2 \lambda r^{2}+10 \lambda r M-9 r M+27 M^{2}\right)}{(\lambda+1)(r-2 M)(\lambda r+3 M)^{2}} A_{\ell m}^{(0)} .
\end{aligned}
$$

From Eq. (A.9) and the expressions for $\partial_{t} K^{\ell m}$ and $\partial_{t} H_{2}^{\ell m}$ in terms of $\partial_{t} \psi_{\text {even }}^{\ell m}$, we find the $H_{1}^{\ell m}$ metric coefficient in the Regge-Wheeler gauge

$$
\begin{aligned}
& H_{1}^{\ell m}=r \partial_{r}\left(\partial_{t} \psi_{\text {even }}^{\ell m}\right)+\frac{\lambda r^{2}-3 M \lambda r-3 M^{2}}{(r-2 M)(\lambda r+3 M)} \partial_{t} \psi_{\text {even }}^{\ell m} \\
& -\frac{8 \pi r^{5}}{(\lambda+1)(r-2 M)(\lambda r+3 M)} \partial_{t} A_{\ell m}^{(0)}+\frac{4 \sqrt{2} i \pi r^{2}}{(\lambda+1)} A_{\ell m}^{(1)} .
\end{aligned}
$$

These equations together with

$$
H_{0}^{\ell m}=H_{2}^{\ell m}+\frac{16 \pi r^{2} F_{\ell m}}{\sqrt{2 \lambda(\lambda+1)}}
$$


give us all metric perturbations on the chosen hypersurface in terms only of $\psi_{\text {even }}^{\ell m}$ and $\partial_{t} \psi_{\text {even }}^{\ell m}$ (and the source). (See also Ref. [14] for general source expressions)

For the specific case of interest of a pointlike particle we have

$$
\begin{aligned}
& K^{\ell m}=\frac{6 M^{2}+3 M \lambda r+\lambda(\lambda+1) r^{2}}{r^{2}(\lambda r+3 M)} \psi_{\text {even }}^{\ell m}+\left(1-\frac{2 M}{r}\right) \partial_{r} \psi_{\text {even }}^{\ell m} \\
& -\frac{8 \pi m_{0} \bar{Y}_{\ell m}(t) U^{0}(t)(r-2 M)^{2}}{(\lambda+1)(\lambda r+3 M) r} \delta[r-R(t)] . \\
& H_{2}^{\ell m}=-\frac{9 M^{3}+9 \lambda M^{2} r+3 \lambda^{2} M r^{2}+\lambda^{2}(\lambda+1) r^{3}}{r^{2}(\lambda r+3 M)^{2}} \psi_{\text {even }}^{\ell m} \\
& +\frac{3 M^{2}-\lambda M r+\lambda r^{2}}{r(\lambda r+3 M)} \partial_{r} \psi_{\text {even }}^{\ell m}+(r-2 M) \partial_{r}^{2} \psi_{\text {even }}^{\ell m} \\
& +\frac{8 \pi m_{0} \bar{Y}_{\ell m}(t) U^{0}(t)\left(1-\frac{2 M}{r}\right)\left[\lambda^{2} r^{2}+2 \lambda M r-3 M r+3 M^{2}\right]}{(\lambda+1)(\lambda r+3 M)^{2}} \delta[r-R(t)] \\
& -\frac{8 \pi m_{0} \bar{Y}_{\ell m}(t) U^{0}(t)(r-2 M)^{2}}{(\lambda+1)(\lambda r+3 M)} \delta^{\prime}[r-R(t)] \text {. } \\
& H_{1}^{\ell m}=r \partial_{r}\left(\partial_{t} \psi_{\text {even }}^{\ell m}\right)+\frac{\lambda r^{2}-3 M \lambda r-3 M^{2}}{(r-2 M)(\lambda r+3 M)} \partial_{t} \psi_{\text {even }}^{\ell m} \\
& -\frac{8 \pi m_{0} \bar{Y}_{\ell m}(t) U^{0}(t) \dot{r}_{p}(\lambda r+M)}{(\lambda+1)(\lambda r+3 M)} \delta[r-R(t)] \\
& +\frac{8 \pi m_{0} \bar{Y}_{\ell m}(t) U^{0}(t) \dot{r}_{p} r(r-2 M)}{(\lambda+1)(\lambda r+3 M)} \delta^{\prime}[r-R(t)] . \\
& -\frac{8 \pi m_{0}\left(d \bar{Y}_{\ell m} / d t\right)(r-2 M) r U^{0}(t)}{(\lambda+1)(\lambda r+3 M)} \delta[r-R(t)] .
\end{aligned}
$$

and

$$
H_{0}^{\ell m}=H_{2}^{\ell m}+16 \pi r^{2} m_{0} U^{0}(t) \operatorname{ang} 1(t) \delta[r-R(t)]
$$

where

$$
\begin{aligned}
& \operatorname{ang} 1(t)=\frac{1}{2}\left[\left(\frac{d \Theta}{d t}\right)^{2}-\sin ^{2} \Theta\left(\frac{d \Phi}{d t}\right)^{2}\right] \bar{W}^{\ell m}+\frac{d \Phi}{d t} \frac{d \Theta}{d t} \bar{X}^{\ell m}, \\
& \bar{X}^{\ell m}=2 \partial_{\varphi}\left(\partial_{\theta}-\cot \theta\right) \bar{Y}^{\ell m}, \\
& \bar{W}^{\ell m}=\left(\partial_{\theta}^{2}-\cot \theta \partial_{\theta}-\frac{1}{\sin ^{2} \theta} \partial_{\varphi}^{2}\right) \bar{Y}^{\ell m} .
\end{aligned}
$$

\section{Appendix B.2. Odd Parity}

We consider the following waveform in terms of generic metric perturbations in the Regge-Wheeler notation 


$$
\begin{aligned}
\psi_{\text {odd }}^{\ell m}(r, t) & =\frac{r}{\lambda}\left[r^{2} \partial_{r}\left(\frac{h_{0}^{\ell m}(r, t)}{r^{2}}\right)-\partial_{t} h_{1}^{\ell m}(r, t)\right] \\
& =\frac{2 r}{\lambda} \sqrt{1-\frac{2 M}{r}} K_{r \theta} .
\end{aligned}
$$

This waveform is related to the Zerilli's [30] and Moncrief's [19] odd parity waveforms $\psi_{Z e r}^{o d d}=\psi_{M o n}^{\text {odd }}$

$$
\psi_{Z e r}^{o d d}=\frac{\left(1-\frac{2 M}{r}\right)}{r}\left[h_{1}^{\ell m}+\frac{r^{2}}{2} \partial_{r}\left(\frac{h_{2}^{\ell m}}{r^{2}}\right)\right],
$$

by (See Eq. (A.16))

$$
\partial_{t} \psi_{\text {odd }}^{\ell m}=2 \psi_{Z e r}^{\text {odd }}-\frac{8 \pi i r(r-2 M) Q^{\ell m}}{\lambda \sqrt{\lambda+1}},
$$

to the Cunningham et al [1] waveform $\psi_{G}^{\ell m}$ by

$$
\psi_{\text {odd }}^{\ell m}=-2 \frac{(\ell-2) !}{(\ell+2) !} \psi_{G}^{\ell m}=-\frac{1}{2} \frac{\psi_{G}^{\ell m}}{\lambda(\lambda+1)},
$$

and to the Weyl scalar $\Psi_{2}$

$$
\Psi_{2}^{-}=\frac{(\ell+2) !}{8(\ell-2) !} \frac{\psi_{\text {odd }}^{\ell m}}{r^{3}}
$$

[Here we used the Kinnersley tetrad, in the Schwarzschild background, and decomposed $\Psi_{2}$ into spherical harmonics].

One can use the field equations to write the metric perturbation in the ReggeWheeler gauge

$$
\begin{aligned}
& h_{0}^{\ell m}(r, t)=\frac{1}{2}\left(1-\frac{2 M}{r}\right) \partial_{r}\left(r \psi_{\text {odd }}^{\ell m}\right)+\frac{4 \pi r^{3} Q_{\ell m}^{(0)}}{\lambda \sqrt{(\lambda+1)}} \\
& h_{1}^{\ell m}(r, t)=\frac{1}{2} \frac{r}{\left(1-\frac{2 M}{r}\right)} \partial_{t} \psi_{\text {odd }}^{\ell m}+\frac{4 \pi i r^{3} Q_{\ell m}}{\lambda \sqrt{(\lambda+1)}}
\end{aligned}
$$

For a source term represented by a particle the corresponding metric perturbations in the Regge-Wheeler gauge are

$$
\begin{aligned}
h_{0}^{\ell m}(r, t) & =\frac{1}{2}\left(1-\frac{2 M}{r}\right) \partial_{r}\left(r \psi_{\text {odd }}^{\ell m}\right) \\
& +\frac{4 \pi m_{0} r(r-2 M) U^{0}(t) \operatorname{ang}(t) \delta[r-R(t)]}{\lambda(\lambda+1)} \\
h_{1}^{\ell m}(r, t) & =\frac{1}{2} \frac{r}{\left(1-\frac{2 M}{r}\right)} \partial_{t} \psi_{\text {odd }}^{\ell m} \\
& -\frac{4 \pi m_{0} r^{3} U^{0}(t)\left(\frac{d}{d t} R\right) \operatorname{ang}(t) \delta[r-R(t)]}{(r-2 M) \lambda(\lambda+1)},
\end{aligned}
$$

where

$$
\operatorname{ang}(t)=\frac{1}{\sin \Theta}\left(\frac{d \Theta}{d t}\right) \partial_{\varphi} \bar{Y}^{\ell m}(\Theta, \Phi)-\sin \Theta\left(\frac{d \Phi}{d t}\right) \partial_{\theta} \bar{Y}^{\ell m}(\Theta, \Phi),
$$

and $R(t), \Theta, \Phi$ define the trajectory of the orbiting particle in spherical coordinates. 


\section{Appendix C. (Inverse) Chandrasekhar transformations in the time domain}

Chandrasekhar transformations deal with the expressions that relate the waveforms in the metric perturbation picture (Regge-Wheeler and Zerilli's) and the Weyl scalars that describe the curvature perturbations (Teukolsky's). Chandrasekhar [9] found the transformations in the frequency domain. Those expressions can be generalized to the time domain to describe local transformations, and take into account matter terms such as a particle. Below we give the explicit expressions for the transformations (we drop the $(\ell m)$ superscript in the waveforms for the sake of notational simplicity.)

\section{Appendix C.1. From Waveforms to Weyl scalars}

To obtain the Weyl scalars from the waveforms (B.1) and (B.16) for even and odd parity respectively we simply substitute into Eqs. (14), (15), (21), and (22) the expressions (B.5) $-(\underline{B .8})$, and (B.21) $-(\underline{B .22})$ for the metric coefficients in the Regge-Wheeler gauge. The result is

$$
\begin{aligned}
& \psi_{4}^{+}=\frac{1}{16 r} \sqrt{\frac{(\ell-2) !}{(\ell+2) !}}\left\{2 \psi_{, r * r *}^{\text {even }}-2 \psi_{, t r *}^{\text {even }}+W^{+}\left(\psi_{, r *}^{\text {even }}-\psi_{, t}^{\text {even }}\right)-V^{+} \psi^{\text {even }}\right. \\
& +\frac{16 \pi r^{3}}{(\lambda r+3 M)(\lambda+1)}\left(\partial_{t} A_{\ell m}^{(0)}-\partial_{r *} A_{\ell m}^{(0)}\right)-\frac{8 i(r-2 M) \sqrt{2} \pi A_{\ell m}^{(1)}(r, t)}{\lambda+1} \\
& +16 \frac{\pi r\left(\lambda^{2} r^{2}-2 \lambda r^{2}+10 \lambda r M-9 r M+27 M^{2}\right) A_{\ell m}^{(0)}(r, t)}{(\lambda+1)(\lambda r+3 M)^{2}} \\
& \left.-8 \frac{F_{\ell m}(r, t) \sqrt{2} \pi(r-2 M)}{\sqrt{\lambda(\lambda+1)}}\right\} \\
& \psi_{4}^{-}=\frac{-i}{16 r} \sqrt{\frac{(\ell-2) !}{(\ell+2) !}}\left\{2 \psi_{, r * r *}^{\text {odd }}-2 \psi_{, t r *}^{\text {odd }}+W^{-}\left(\psi_{, r *}^{\text {odd }}-\psi_{, t}^{\text {odd }}\right)-V^{-} \psi^{\text {odd }}\right. \\
& -\frac{16 \pi r^{2}}{\lambda(\lambda+1)}\left(\partial_{t} Q_{\ell m}^{(0)}-\partial_{r *} Q_{\ell m}^{(0)}\right)+\frac{16 i \pi r(r-2 M)}{\lambda(\lambda+1)}\left(\partial_{t} Q_{\ell m}-\partial_{r *} Q_{\ell m}\right) \\
& \left.-\frac{48 i \pi(r-2 M)^{2} Q_{\ell m}}{\lambda \sqrt{\lambda+1} r}+\frac{16 \pi(3 r-8 M) Q_{\ell m}^{(0)}}{\lambda \sqrt{\lambda+1}}-S^{-}\right\} \text {, } \\
& \psi_{0}^{+}=\frac{1}{4 f^{2} r} \sqrt{\frac{(\ell-2) !}{(\ell+2) !}}\left\{2 \psi_{, r * r *}^{\text {even }}+2 \psi_{, t r *}^{\text {even }}+W^{+}\left(\psi_{, r *}^{\text {even }}+\psi_{, t}^{\text {even }}\right)-V^{+} \psi^{\text {even }}\right. \\
& -\frac{16 \pi r^{3}}{(\lambda r+3 M)(\lambda+1)}\left(\partial_{t} A_{\ell m}^{(0)}+\partial_{r *} A_{\ell m}^{(0)}\right)+\frac{8 i(r-2 M) \sqrt{2} \pi A_{\ell m}^{(1)}(r, t)}{\lambda+1} \\
& +16 \frac{\pi r\left(\lambda^{2} r^{2}-2 \lambda r^{2}+10 \lambda r M-9 r M+27 M^{2}\right) A_{\ell m}^{(0)}(r, t)}{(\lambda+1)(\lambda r+3 M)^{2}} \\
& \left.-8 \frac{F_{\ell m}(r, t) \sqrt{2} \pi(r-2 M)}{\sqrt{\lambda(\lambda+1)}}\right\}
\end{aligned}
$$


Reconstruction of Black Hole Metric Perturbations from Weyl Curvature II

$$
\begin{aligned}
\psi_{0}^{-}= & \frac{i}{4 f^{2} r} \sqrt{\frac{(\ell-2) !}{(\ell+2) !}}\left\{2 \psi_{, r * r *}^{\text {odd }}+2 \psi_{, t r *}^{\text {odd }}+W^{-}\left(\psi_{, r *}^{\text {odd }}+\psi_{, t}^{\text {odd }}\right)-V^{-} \psi^{\text {odd }}\right. \\
& \frac{16 \pi r^{2}}{\lambda(\lambda+1)}\left(\partial_{t} Q_{\ell m}^{(0)}+\partial_{r *} Q_{\ell m}^{(0)}\right)+\frac{16 i \pi r(r-2 M)}{\lambda(\lambda+1)}\left(\partial_{t} Q_{\ell m}+\partial_{r *} Q_{\ell m}\right) \\
& \left.+\frac{48 i \pi(r-2 M)^{2} Q_{\ell m}}{\lambda \sqrt{\lambda+1} r}+\frac{16 \pi(3 r-8 M) Q_{\ell m}^{(0)}}{\lambda \sqrt{\lambda+1}}-S^{-}\right\}
\end{aligned}
$$

where we introduced the Chandrasekhar notation for

$$
\begin{aligned}
V^{+} & =2\left(1-\frac{2 M}{r}\right) \frac{\left[\lambda^{2}(\lambda+1) r^{3}+3 \lambda^{2} M r^{2}+9 \lambda M^{2} r+9 M^{3}\right]}{r^{3}(\lambda r+3 M)^{2}}, \\
V^{-} & =2\left(1-\frac{2 M}{r}\right)\left(\frac{\lambda+1}{r^{2}}-3 \frac{M}{r^{3}}\right) \\
W^{+} & =2 \frac{\left(\lambda r^{2}-3 \lambda M r-3 M^{2}\right)}{r^{2}(\lambda r+3 M)} \\
W^{-} & =2 \frac{(r-3 M)}{r^{2}}
\end{aligned}
$$

and

$$
S^{-}=-\frac{8 \pi(r-2 M)}{\lambda \sqrt{(\lambda+1)}}\left[\frac{\partial}{\partial r}\left(r Q^{(0)}(r, t)\right)-i r \frac{\partial}{\partial t} Q(r, t)\right],
$$

is the source term for the Regge-Wheeler wave equation.

These relations are local, and in the time domain. Compare to the expressions in the frequency domain of Ref. [9, Eqs. (345) and (353) in Chapter 4.

\section{Appendix C.2. From Weyl scalars to Waveforms}

To obtain the inverse Chandrasekhar relations we make use of Eqs. (16)-(20), and (29)(26) for the metric coefficients entering in the definitions of the even and odd parity 
waveforms, Eqs. (B.1) and (B.16), respectively

$$
\begin{aligned}
& \psi^{\text {even }}=\frac{r^{2}(r-2 M) \frac{\partial^{2}}{\partial r^{2}} H_{0}(r, t)}{\lambda(\lambda+1)}-\frac{r^{3} \frac{\partial^{2}}{\partial r \partial t} H_{1}(r, t)}{\lambda(\lambda+1)} \\
& +\frac{r\left(r M \lambda-3 M^{2}+r^{2} \lambda+6 r M\right) \frac{\partial}{\partial r} H_{0}(r, t)}{(\lambda+1) \lambda(\lambda r+3 M)} \\
& +\frac{\left(2 r^{2} \lambda-5 r M \lambda-21 M^{2}+9 r M\right) r^{2} \frac{\partial}{\partial t} H_{1}(r, t)}{(\lambda+1)(\lambda r+3 M)(-r+2 M) \lambda} \\
& -\frac{\left(M^{2} \lambda r+2 r^{2} M \lambda^{2}-12 r^{2} M-r^{2} M \lambda-2 r^{3} \lambda+42 r M^{2}-r^{3} \lambda^{2}-63 M^{3}\right) H_{0}(r, t)}{2(\lambda+1)(\lambda r+3 M)(-r+2 M) \lambda} \\
& -4 \frac{r^{2} \pi \sqrt{2 \lambda+2}\left(-5 r^{2} \lambda-12 r M+9 r M \lambda+21 M M^{2}\right) \sqrt{2} B(r, t)}{(\lambda+1)^{2} \lambda(\lambda r+3 M)} \\
& +\frac{r^{4} A^{(0)}(r, t)}{\lambda(\lambda+1)(-r+2 M)} \\
& +4 \frac{\sqrt{2} \sqrt{\lambda(\lambda+1)} r^{2} \pi\left(2 r M-11 M^{2}-r^{2} \lambda+2 r M \lambda\right) F(r, t)}{(-r+2 M)(\lambda+1)^{2} \lambda^{2}} \\
& -8 \frac{r^{3}(-r+2 M) \pi \frac{\partial}{\partial r} B(r, t)}{(\lambda+1)^{3 / 2} \lambda}-8 \frac{r^{3} M \pi \sqrt{2} \frac{\partial}{\partial r} F(r, t)}{(\lambda+1) \sqrt{\lambda(\lambda+1)} \lambda},
\end{aligned}
$$

and

$$
\psi^{\text {odd }}=\frac{r}{\lambda}\left\{-\frac{2}{r} \frac{\left(1-\frac{M}{r}\right)}{\left(1-\frac{2 M}{r}\right.} h_{0}+S_{0}-\partial_{t} h_{1}\right\} .
$$

So, finally

$$
\begin{aligned}
\psi^{\text {odd }}= & \frac{r}{\lambda}\left\{-\frac{2}{r}\left(1-\frac{M}{r}\right) \int_{2 M}^{r} \frac{S_{0}\left(r^{\prime}, t\right)}{1-\frac{2 M}{r^{\prime}}} d r^{\prime}+\frac{4 i r^{2}}{f} \sqrt{\frac{(\ell+2) !}{(\ell-2) !}}\left(\psi_{4}^{-}-\frac{f^{2}}{4} \psi_{0}^{-}\right)\right. \\
& \left.-\frac{2}{\sqrt{1-\frac{2 M}{r}}}\left[\int_{2 M}^{r} \partial_{t} S_{1}\left(r^{\prime}, t\right) \sqrt{1-\frac{2 M}{r^{\prime}}} d r^{\prime}\right]\right\} .
\end{aligned}
$$

\section{Appendix D. Symmetric tetrad}

A further algebraic simplification of the expressions can be achieved by choosing the background tetrad such that we treat $\psi_{4}$ and $\psi_{0}$ on the same footing, thus allowing simple linear combinations of the sort $\psi_{4}^{S} \pm \psi_{0}^{S}$ in the expression in Section 3. The components of the symmetric null tetrad for the Kerr background are given by

$$
\begin{aligned}
& \left(\mathrm{l}_{\mathrm{S}}{ }^{\alpha}\right)=\left(\frac{r^{2}+a^{2}}{\sqrt{2 \Delta \Sigma}}, \sqrt{\frac{\Delta}{2 \Sigma}}, 0, \frac{a}{\sqrt{2 \Delta \Sigma}}\right), \\
& \left(\mathrm{n}_{\mathrm{S}}{ }^{\alpha}\right)=\left(\frac{r^{2}+a^{2}}{\sqrt{2 \Delta \Sigma}},-\sqrt{\frac{\Delta}{2 \Sigma}}, 0, \frac{a}{\sqrt{2 \Delta \Sigma}}\right), \\
& \left(\mathrm{m}_{\mathrm{S}}{ }^{\alpha}\right)=\frac{1}{\sqrt{2}(r+i a \cos \theta)}\left(i a \sin \theta, 0,1, \frac{i}{\sin \theta}\right) .
\end{aligned}
$$


With the above choice of the tetrad the spin coefficients are

$$
\begin{aligned}
& \nu_{S}=, \quad \sigma_{S}=, \quad \lambda_{S}=, \quad \kappa_{S}=0, \\
& \pi_{S}=\pi_{K}=i a \rho_{K}^{2} \frac{\sin \theta}{\sqrt{2}}, \\
& \tau_{S}=\tau_{K}=-i a \rho_{K} \bar{\rho}_{K} \frac{\sin \theta}{\sqrt{2}}, \\
& \rho_{S}=\mu_{S}=\sqrt{\frac{\Delta}{2 \Sigma}} \rho_{K} \\
& \epsilon_{S}=\frac{\left[M\left(r^{2}-a^{2} \cos ^{2} \theta\right)-a^{2} r \sin ^{2} \theta\right]}{2 \sqrt{2 \Delta \Sigma^{3}}} \\
& \gamma_{S}=\epsilon_{S}-i a \cos \theta \sqrt{\frac{\Delta}{2 \Sigma^{3}}} \\
& \alpha_{S}=\frac{\left[\left(r^{2}+a^{2}\right) \cos \theta-2 i a r \sin ^{2} \theta\right]}{2 \sqrt{2} \sin \theta}\left(\rho_{K}^{2} \bar{\rho}_{K}\right) \\
& \beta_{S}=-\frac{\left[\left(r^{2}+a^{2}\right) \cot \theta\right]}{2 \sqrt{2}}\left(\bar{\rho}_{K}^{2} \rho_{K}\right), \quad \alpha_{S}-\bar{\beta}_{S}=\alpha_{K}-\bar{\beta}_{K},
\end{aligned}
$$

where an overbar stands for complex conjugation.

The Weyl scalars computed with the Symmetric tetrad relate to those computed with the Kinnersley tetrad as follows

$$
\begin{aligned}
& \psi_{4}^{S}=\left(\frac{2 \Sigma}{\Delta}\right) \psi_{4}^{K}, \quad \psi_{3}^{S}=\sqrt{\frac{2 \Sigma}{\Delta}} \psi_{3}^{K}, \quad \psi_{2}^{S}=\psi_{2}^{K}, \\
& \psi_{1}^{S}=\sqrt{\frac{\Delta}{2 \Sigma}} \psi_{1}^{K}, \quad \psi_{0}^{S}=\left(\frac{\Delta}{2 \Sigma}\right) \psi_{0}^{K} .
\end{aligned}
$$

\section{References}

[1] J. Baker, B. Brügmann, Manuela Campanelli, C. O. Lousto, and R. Takahashi, Plunge waveforms from inspiralling binary black holes, Phys. Rev. Lett. 87 (2001), 121103.

[2] J. Baker, M. Campanelli, C. O. Lousto, and R. Takahashi, Modeling gravitational radiation from coalescing binary black holes, Phys. Rev. D 65 (2002), 124012.

[3] — Coalescence remnant of spinning binary black holes, Phys. Rev. D 69 (2004), 027505.

[4] John Baker, Bernd Brügmann, Manuela Campanelli, and Carlos O. Lousto, Gravitational waves from black hole collisions via an eclectic approach, Class. Quantum Grav. 17 (2000), L149-L156.

[5] John Baker, Manuela Campanelli, and Carlos O. Lousto, The Lazarus project: A pragmatic approach to binary black hole evolutions, Phys. Rev. D 65 (2002), 044001.

[6] Leor Barack and Carlos O. Lousto, Computing the gravitational self-force on a compact object plunging into a schwarzschild black hole, Phys. Rev. D 66 (2002), 061502.

[7] Leor Barack and Amos Ori, Gravitational self force and gauge transformations, Phys. Rev. D 64 (2001), 124003.

[8] M. Campanelli and C. O. Lousto, Second order gauge invariant gravitational perturbations of a Kerr black hole, Phys. Rev. D 59 (1999), 124022.

[9] S. Chandrasekhar, The mathematical theory of black holes, Oxford University Press, Oxford, England, 1983. 
[10] P. L. Chrzanowski, Vector potential and metric perturbations of a rotating black hole, Phys. Rev. D 11 (1975), 2042-2062.

[11] C. T. Cunningham, R. H. Price, and V. Moncrief, Radiation from collapsing relativistic stars. I. Linearized odd-parity radiation, Astrophys. J. 224 (1978), 643.

[12] Steven Detweiler and Eric Poisson, Low multipole contributions to the gravitational self- force, Phys. Rev. D 69 (2004), 084019.

[13] R. Giacconi and R. Ruffini (eds.), Physics and astrophysics of neutron stars and black holes, 1978.

[14] Sanjay Jhingan and Takahiro Tanaka, Improvement on the metric reconstruction scheme in reggewheeler-zerilli formalism, Phys. Rev. D 67 (2003), 104018.

[15] L. S. Kegeles and J. M. Cohen, Constructive procedure for perturbations of space-times, Phys. Rev. D 19 (1979), 1641-1664.

[16] Carlos O. Lousto, Pragmatic Approach to Gravitational Radiation Reaction in Binary Black Holes, Phys. Rev. Lett. 84 (2000), 5251.

[17] Carlos O. Lousto and Richard H. Price, Understanding initial data for black hole collisions, Phys. Rev. D 56 (1997), 6439-6457.

[18] Carlos O. Lousto and Bernard F. Whiting, Reconstruction of black hole metric perturbations from weyl curvature, Phys. Rev. D 66 (2002), 024026.

[19] V. Moncrief, Gravitational perturbations of spherically symmetric systems. I. the exterior problem, Annals of Physics 88 (1974), 323-342.

[20] E. Newman and R. Penrose, An approach to gravitational radiation by a method of spin coefficients, J. Math. Phys. 3 (1962), 566.

[21] Amos Ori, Reconstruction of inhomogeneous metric perturbations and electromagnetic fourpotential in kerr spacetime, Phys. Rev. D 67 (2003), 124010.

[22] T. Regge and J. Wheeler, Stability of a Schwarzschild singularity, Phys. Rev. 108 (1957), no. 4, 1063-1069.

[23] Norichika Sago, Hiroyuki Nakano, and Misao Sasaki, Gauge problem in the gravitational self-force. i: Harmonic gauge approach in the schwarzschild background, Phys. Rev. D 67 (2003), 104017.

[24] J. M. Stewart, Hertz-Bromwich-Debye-Whittaker-Penrose potentials in general relativity, Royal Society of London Proceedings Series A 367 (1979), 527-538.

[25] S. A. Teukolsky, Perturbations of a rotating black hole. I. fundamental equations for gravitational, electromagnetic, and neutrino-field perturbations, Astrophys. J. 185 (1973), 635-647.

[26] R. M. Wald, On perturbations of a kerr black hole, J. Math. Phys. 14 (1973), 1453.

[27] R. M. Wald, Construction of metric and vector potential perturbations of a Reissner-Nordstrom black hole, Royal Society of London Proceedings Series A 369 (1979), 67-81.

[28] Bernard F. Whiting and Larry R. Price, Metric reconstruction from weyl scalars, Class. Quantum Grav. 22, S589(2005).

[29] F. J. Zerilli, Effective potential for even-parity Regge-Wheeler gravitational perturbation equations, Phys. Rev. Lett. 24 (1970), no. 13, 737-738.

[30] _ Gravitational field of a particle falling in a Schwarzschild geometry analyzed in tensor harmonics, Phys. Rev. D. 2 (1970), 2141. 